\title{
The impact of $\alpha /$ Fe enhanced stellar evolutionary tracks on the ages of elliptical galaxies
}

\author{
D. Thomas and C. Maraston
}

\author{
Max-Planck-Institut für extraterrestrische Physik, Giessenbachstraße, 85748 Garching, Germany \\ e-mail: dthomas@mpe.mpg.de; maraston@mpe.mpg.de
}

Received 16 September 2002 / Accepted 30 January 2003

\begin{abstract}
We complement our study of $\alpha / F e$ enhanced stellar population models of Lick absorption indices (Thomas et al. 2003) by comparing two sets of $\alpha / \mathrm{Fe}$ enhanced models. In both models the impact on Lick indices due to $\alpha / \mathrm{Fe}$ enhancement is accounted for through a modification of the stellar absorption line-strengths using the response functions of Tripicco \& Bell (1995). One set of models, however, uses solar-scaled, the other $\alpha / \mathrm{Fe}$ enhanced stellar evolutionary tracks. Since the $\alpha / \mathrm{Fe}$ enhanced tracks are hotter than the solar-scaled ones (Salasnich et al. 2000), the correspondent stellar population models have slightly weaker metallic indices (i.e. $\mathrm{Mg} b$, $\langle\mathrm{Fe}\rangle$ etc.) and stronger Balmer line indices $(\mathrm{H} \beta)$ (Maraston et al. 2003). Here we explore quantitatively the impact of this effect on the $\alpha / \mathrm{Fe}$ ratios, metallicities and ages that are derived for elliptical galaxies. We find that the modest decrease of the metallic indices $\mathrm{Mg} b$ and $\langle\mathrm{Fe}\rangle$ balance each other, such that fully consistent $\alpha / \mathrm{Fe}$ ratios are derived for stellar systems using $\alpha / \mathrm{Fe}$ enhanced models with either solar-scaled or $\alpha / \mathrm{Fe}$ enhanced stellar tracks. The decrease of the metallic indices and the increase of $\mathrm{H} \beta$ conspire in a way that also consistent metallicities are obtained. The derived ages, instead, are significantly different. The inclusion of $\alpha / \mathrm{Fe}$ enhanced stellar tracks leads to the derivation of ages as high as $\sim 30 \mathrm{Gyr}$ for elliptical galaxies. For the same objects, ages not older than $15 \mathrm{Gyr}$ are obtained, if $\alpha / \mathrm{Fe}$ enhanced models using solar-scaled tracks are adopted. This may indicate that current stellar evolutionary models overestimate the bluing of stellar evolutionary tracks due to $\alpha /$ Fe enhanced chemical mixtures at super-solar metallicities.
\end{abstract}

Key words. stars: evolution - stars: abundances - galaxies: stellar content - galaxies: elliptical and lenticular, cD galaxies: formation

\section{Introduction}

The analysis of elliptical galaxy absorption lines in the early $90 \mathrm{~s}$ revealed an inadequacy of standard models to recover the $\mathrm{Mg}$ and Fe Lick indices measured in these galaxies. The failure was attributed to element abundance ratios different from solar. More specifically, an enhanced ratio of $\alpha$-elements (e.g. $\mathrm{Mg}, \mathrm{O}, \mathrm{Ca}$, etc.) with respect to iron was the most straightforward interpretation (Worthey et al. 1992). Recently an accurate model calibration with metal-rich globular clusters has confirmed the early suggestion (Maraston et al. 2003). In fact, standard stellar population models are found to be unable to recover the indices also of Bulge globular clusters that are known, from high-resolution spectroscopy of individual stars, to have enhanced $\alpha / \mathrm{Fe}$ ratios. These findings call clearly for an update of the standard models, in particular for the inclusion of $\alpha / \mathrm{Fe}$ enhancement.

Therefore, in Thomas et al. (2003, hereafter TMB) we present the Lick absorption indices of simple stellar population models (SSP) which, for the first time, are computed for variable element abundance ratios, in particular $\alpha / \mathrm{Fe}$. The key ingredient in the models is the response of absorption lines to

Send offprint requests to: D. Thomas, e-mail: dthomas@mpe.mpg. de the abundance variations of individual elements in the stellar atmospheres. This is the major effect to be taken into account (see also Trager et al. 2000). The secondary effect of $\alpha /$ Fe enhancement on SSP models comes from the adopted stellar evolutionary tracks, since the element abundance variations in a star affect also the star's evolution and opacities, hence the effective temperature. This secondary effect has been neglected in the TMB models, because they are based on the SSPs of Maraston (1998), which use solar-scaled tracks (Cassisi et al. 1997). However, the TMB models are calibrated with globular clusters and reproduce very well their metallicities and $\alpha / \mathrm{Fe}$ ratios in the metallicity range up to solar. This does not come as a surprise, since the difference between $\alpha / \mathrm{Fe}$ enhanced and solarscaled stellar evolutionary tracks is negligible at low metallicities (Salaris \& Weiss 1998; Salasnich et al. 2000; VandenBerg et al. 2000; Kim et al. 2002).

A larger difference is found at super-solar metallicities (Salasnich et al. 2000). More specifically, $\alpha /$ Fe enhanced stellar tracks have hotter turnoffs and hotter giant branches owing to the lower opacities of $\alpha / \mathrm{Fe}$ enhanced stellar atmospheres (Salasnich et al. 2000; VandenBerg et al. 2000; Kim et al. 2002). Maraston et al. (2003) explore the effect on SSP models. They show that SSPs using $\alpha / \mathrm{Fe}$ enhanced tracks have 
moderately weaker metallic (e.g., $\mathrm{Mg} b,\langle\mathrm{Fe}\rangle$ ) indices, and significantly stronger Balmer $(\mathrm{H} \beta)$ indices for the same age and metallicity. The aim of this paper is to estimate this effect on the TMB models, and to discuss the impact on the ages, metallicities, and $\alpha / \mathrm{Fe}$ ratios derived for elliptical galaxies.

The paper is organized as follows. Section 2 gives a brief overview of the development and current status of $\alpha / \mathrm{Fe}$ enhanced stellar evolutionary tracks. The modified TMB models are described in Sect. 3 and compared with elliptical galaxy data in Sect. 4. The results are summarized and discussed in Sect. 5.

\section{A brief history of $\alpha / \mathrm{Fe}$ enhanced stellar tracks}

The development of stellar evolutionary tracks with chemical mixtures different from the solar proportions has made major progress in the last years. Based on the assumption that the abundance ratio of elements with high ionisation potential (HPE: C, N, O, Ne) to the elements with low ionisation potential (LPE: $\mathrm{Mg}, \mathrm{Si}, \mathrm{S}, \mathrm{Ca}, \mathrm{Fe}$ ) remains constant relative to the solar ratio in an $\alpha / \mathrm{Fe}$ enhanced isochrone, Salaris et al. (1993) find that an $\alpha / F e$ enhanced isochrone is cooler than the solar-scaled one and can be mimicked by a solar-scaled isochrone with higher metallicity. The reason for the validity of this metallicity-scaling is that the HPE control the temperature of the turnoff, while the LPE impact on the red giant branch (see also discussion in Trager et al. 2000), so that the constancy of the HPE/LPE ratio ensures the shape of the stellar evolutionary track not to vary.

But the ratio of HPE to LPE is actually super-solar for realistic $\alpha / \mathrm{Fe}$ enhanced mixtures, and the shape of the isochrone is not conserved (Weiss et al. 1995). Most importantly, Salaris $\&$ Weiss $(1998)$ find that for $[\mathrm{HPE} / \mathrm{LPE}] \approx 0.1$ the turnoff and also the red giant branch of an $\alpha / \mathrm{Fe}$ enhanced isochrone are bluer than that of a solar-scaled one, in contrast to the results of Salaris et al. (1993). However, the study of Salaris \& Weiss (1998) is restricted to sub-solar metallicities. Recently, Salasnich et al. (2000) published a set of $\alpha / \mathrm{Fe}$ enhanced $([\alpha / \mathrm{Fe}]=0.3)$ stellar tracks for super-solar metallicities. The authors confirm the conclusion found by Salaris \& Weiss (1998) at low metallicities: "at the same evolutionary stage, the solar-scaled track is fainter, cooler, and older than the $\alpha$-enhanced one" (Salasnich et al. 2000). VandenBerg et al. (2000) and Kim et al. (2002) come to the same conclusion.

\section{The modified TMB model}

We consider the synthetic Lick indices of three different sets of SSP models (see Table 1).

1. The TMB model for $[\alpha / \mathrm{Fe}]=0.3($ Model 1$)$.

2. A modification of Model 1 that includes additionally the effect of $\alpha / \mathrm{Fe}$ enhanced stellar evolutionary tracks (Model 2).

3. The standard solar-scaled $([\alpha / \mathrm{Fe}]=0.0)$ model (Model 3).

Model 1 and Model 2 have the enhanced $\alpha / \mathrm{Fe}$ ratio $[\alpha / \mathrm{Fe}]=$ 0.3. In both, the element abundance effects on the strength of the absorption features, i.e. on the Lick indices, are taken into
Table 1. The three model flavors.

\begin{tabular}{cccc}
\hline \hline & & \multicolumn{2}{c}{ Effect of $\alpha /$ Fe on } \\
& {$[\alpha / \mathrm{Fe}]$} & Absorption Lines & Stellar Tracks \\
\hline Model 1 & 0.3 & yes & no \\
Model 2 & 0.3 & yes & yes \\
Model 3 & 0.0 & no & no \\
\hline
\end{tabular}

account as described in detail in TMB. The impact of $\alpha /$ Fe enhancement on the underlying stellar evolutionary tracks, instead, is neglected in Model 1 but considered in Model 2 as explained in the following.

In both models the stellar evolutionary tracks (solar-scaled) are taken from Cassisi et al. (1997) and S. Cassisi (1999, private communication). As these authors do not yet provide stellar tracks for non-solar element ratios, in Model 2 we mimic the effect of $\alpha / \mathrm{Fe}$ enhancement on the stellar tracks by means of the work of Salasnich et al. (2000). Their stellar tracks are used in a differential way in order to be independent of intrinsic differences with respect to the stellar tracks of Cassisi et al. (1997). More specifically, we use the fluxes in the lines and in the continua of the SSP models of Maraston et al. (2003) computed with the solar-scaled and the $\alpha / \mathrm{Fe}$ enhanced stellar tracks of Salasnich et al. (2000), in order to determine the fractional flux changes caused by the $\alpha / \mathrm{Fe}$ enhanced stellar tracks. Model 2 is then obtained by applying these fractional changes to the fluxes in the lines and continua of Model 1.

\section{Comparison with elliptical galaxies}

In this section we compare the Lick indices $\mathrm{H} \beta, \mathrm{Mg} b$, and $\langle\mathrm{Fe}\rangle$ of the three model flavors of Table 1 with observational data of early-type galaxies.

The data sample comprises 126 objects, 71 of which are field and 55 cluster objects, containing roughly equal fractions of elliptical and lenticular (S0) galaxies. The sample is constructed from the following sources: 41 Virgo cluster and field galaxies (González 1993), 32 Coma cluster galaxies (Mehlert et al. 2000), and 53 mostly field galaxies (the highest quality data from Beuing et al. 2002) selected from the ESO-LV catalog (Lauberts \& Valentijn 1989). For sake of homogeneity, in all cases the central line-strength indices within $0.1 r_{\mathrm{e}}$ are considered. A detailed assessment of the stellar population parameters of the data and the discussion in the context of galaxy formation is given in Thomas et al. (2002).

\section{1. $\alpha /$ Fe ratios}

Figure 1 shows Model 1 and Model 2 for various ages and metallicities as dashed and solid lines in the $\mathrm{Mg} b-\langle\mathrm{Fe}\rangle$ plane. Grey circles are the early-type galaxy data quoted above. Black squares are the two Bulge clusters NGC 6528 and NGC 6553 from Puzia et al. (2002).

The inclusion of $\alpha / \mathrm{Fe}$ enhanced stellar tracks (Model 2) has only a negligible impact on the models in the $\mathrm{Mg} b-\langle\mathrm{Fe}\rangle$ plane. This is consistent with the conclusion of Maraston et al. (2003) for the solar-scaled models that the decrease of metallic line strengths like $\mathrm{Mg} b$ and $\langle\mathrm{Fe}\rangle$ due to the $\alpha / \mathrm{Fe}$ enhancement 


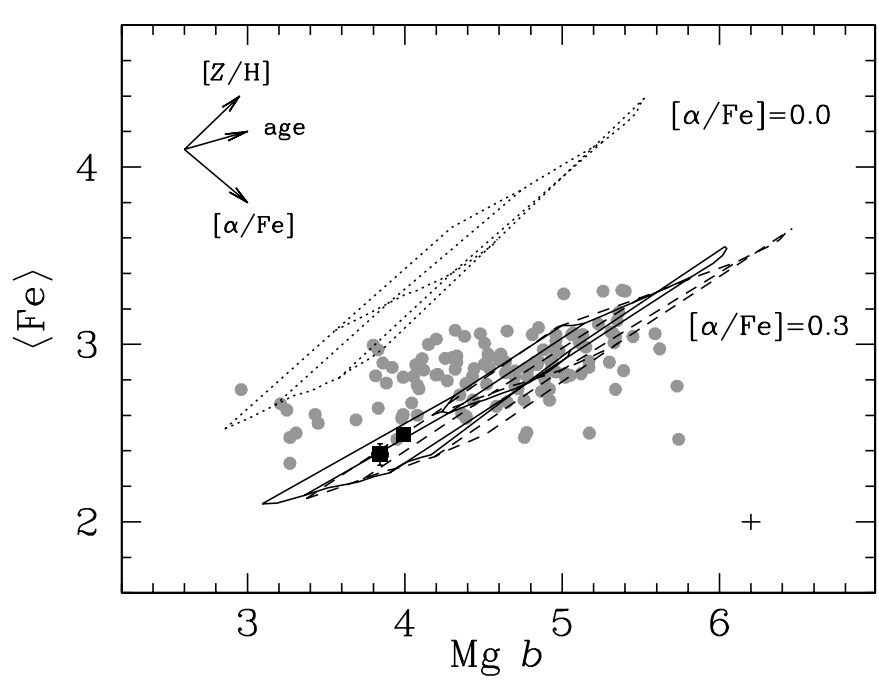

Fig. 1. $\mathrm{Mg} b$ vs. $\langle\mathrm{Fe}\rangle$ for SSP models of constant ages $t=$ $3,5,10,15 \mathrm{Gyr}$ and metallicities $[\mathrm{Z} / \mathrm{H}]=0.0,0.35,0.67$. The dashed and solid lines are the $\alpha / \mathrm{Fe}$ enhanced models Model 1 and Model 2, the dotted lines are Model 3 (see Table 1 and Sect. 3). Grey circles are early-type galaxy data measured within $0.1 r_{\mathrm{e}}$ (González 1993; Mehlert et al. 2000; Beuing et al. 2002). The typical error is given in the lower-right corner. Black squares are the Bulge clusters NGC 6528 and NGC 6553 (Puzia et al. 2002).

of the stellar tracks is comparable. This ensures that consistent $\alpha / \mathrm{Fe}$ ratios are derived irrespective of whether we adopt Model 1 or Model 2.

For comparison, the solar-scaled model (Model 3) is shown as dotted lines in Fig. 1. It becomes obvious that the major effect of element abundance variations is the direct response of the absorption lines, as accounted for in the TMB models (Model 1).

\subsection{Ages and metallicities}

In Fig. 2, $\mathrm{Mg} b$ and $\langle\mathrm{Fe}\rangle$ of Model 1 and Model 2 are plotted vs. $\mathrm{H} \beta$. Consistent with Maraston et al. (2003), Model 2 has significantly stronger $\mathrm{H} \beta$ lines (by up to $\sim 0.26 \AA$ at $15 \mathrm{Gyr}$ ) than Model 1, because of the hotter turnoffs of the $\alpha / \mathrm{Fe}$ enhanced stellar tracks. The strengthening of $\mathrm{H} \beta$ conspires with the weakening of the metallic lines such that going from Model 1 to Model 2 the grid moves along the lines of constant metallicity. Apparently, in current stellar evolution models enhancing the $\alpha / \mathrm{Fe}$ element ratio and decreasing the age have very similar effects on a stellar isochrone. This implies that the same metallicities are derived from Model 1 and Model 2.

In contrast, ages are severely affected. Model 2 predicts significantly stronger $\mathrm{H} \beta$ indices at the same age and metallicity, which leads to the derivation of considerably older ages. The decision whether Model 1 or Model 2 is more appropriate relies on the calibration with globular clusters for which ages and element abundances are independently known. The ages derived from Balmer lines for the two solar metallicity Bulge globular clusters NGC 6528 and NGC 6553 with SSP models using $\alpha / \mathrm{Fe}$ enhanced stellar tracks are in better agreement with the ages inferred from color-magnitude diagrams (Maraston et al. 2003). This is visualized in Fig. 2 with the globular clusters being the filled squares. However, the uncertainties are too large to allow for a firm conclusion in favor of Model 2.

Since the relative increase of the $\mathrm{H} \beta$ line-strength due to $\alpha / \mathrm{Fe}$ enhanced tracks increases with metallicity, the discrepancy between the ages obtained with Model 1 and Model 2 is more pronounced for elliptical galaxies than for the most metal-rich globular clusters. A considerable fraction of the data lies significantly below the 15 Gyr line of Model 2 (Fig. 2). Model 2 yields ages up to $\sim 30$ Gyr (in extrapolation), while with Model 1 we obtain ages not older than $\sim 15$ Gyr for the same data set.

\section{Discussion and conclusion}

We complement our study of $\alpha / \mathrm{Fe}$ enhanced stellar population models of Lick absorption indices (Thomas et al. 2003, TMB) by comparing two sets of $\alpha / \mathrm{Fe}$ enhanced models. In both models the major effect from $\alpha / \mathrm{Fe}$ enhancement on Lick indices is accounted for through a modification of the stellar absorption line-strengths using the response functions of Tripicco $\&$ Bell (1995). One set of models, however, uses solar-scaled (Model 1, equivalent to the TMB model), the other $\alpha / \mathrm{Fe}$ enhanced (Model 2) stellar evolutionary tracks. Here we compare the $\alpha / \mathrm{Fe}$ ratios, metallicities and ages that are derived for elliptical galaxies from Model 1 and Model 2.

Because of the hotter red giant branches of the $\alpha / \mathrm{Fe}$ enhanced stellar tracks, Model 2 has weaker metallic index line-strengths than Model 1. The hotter turnoffs of the $\alpha / \mathrm{Fe}$ enhanced stellar tracks lead to considerably larger Balmer line indices $(\mathrm{H} \beta)$ in Model 2. Both $\mathrm{Mg} b$ and $\langle\mathrm{Fe}\rangle$ indices are reduced almost equally, so that the determination of $\alpha / \mathrm{Fe}$ ratios for stellar systems is not significantly affected. The decrease of the metallic indices and the increase of $\mathrm{H} \beta$ conspire in a way that also consistent metallicities are obtained with Model 1 and Model 2.

The age derivation for metal-rich stellar populations, instead, is severely affected by the use of $\alpha / \mathrm{Fe}$ enhanced stellar tracks. The stronger $\mathrm{H} \beta$ lines of Model 2 with respect to Model 1 imply significantly older ages. The higher the metallicity the more pronounced is this increase of $\mathrm{H} \beta$ in Model 2, and average ages up to $\sim 30 \mathrm{Gyr}$ are obtained for early-type galaxies.

Such high ages appear larger than current estimates of the age of the universe ( 15 Gyr, Ferreras et al. 2001) as constrained by cosmic microwave background (Knox et al. 2001) and high-redshift supernova (Riess et al. 1998; Perlmutter et al. 1999) measurements. This may indicate that the bluing of the stellar tracks due to $\alpha /$ Fe enhanced chemical mixtures at supersolar metallicities is overestimated in current stellar evolution models.

The key check, whether $\alpha / \mathrm{Fe}$ enhancement is described adequately in the stellar evolutionary tracks, is the comparison with globular cluster data. However, at metallicities well below solar, where plenty of data of globular clusters are available, the effect of $\alpha / \mathrm{Fe}$ enhancement on stellar evolution is negligible. Around solar metallicity the effect is appreciable, but the presently available calibrations do not provide a clear answer. The comparison with the color-magnitude-diagram of 47 Tuc 


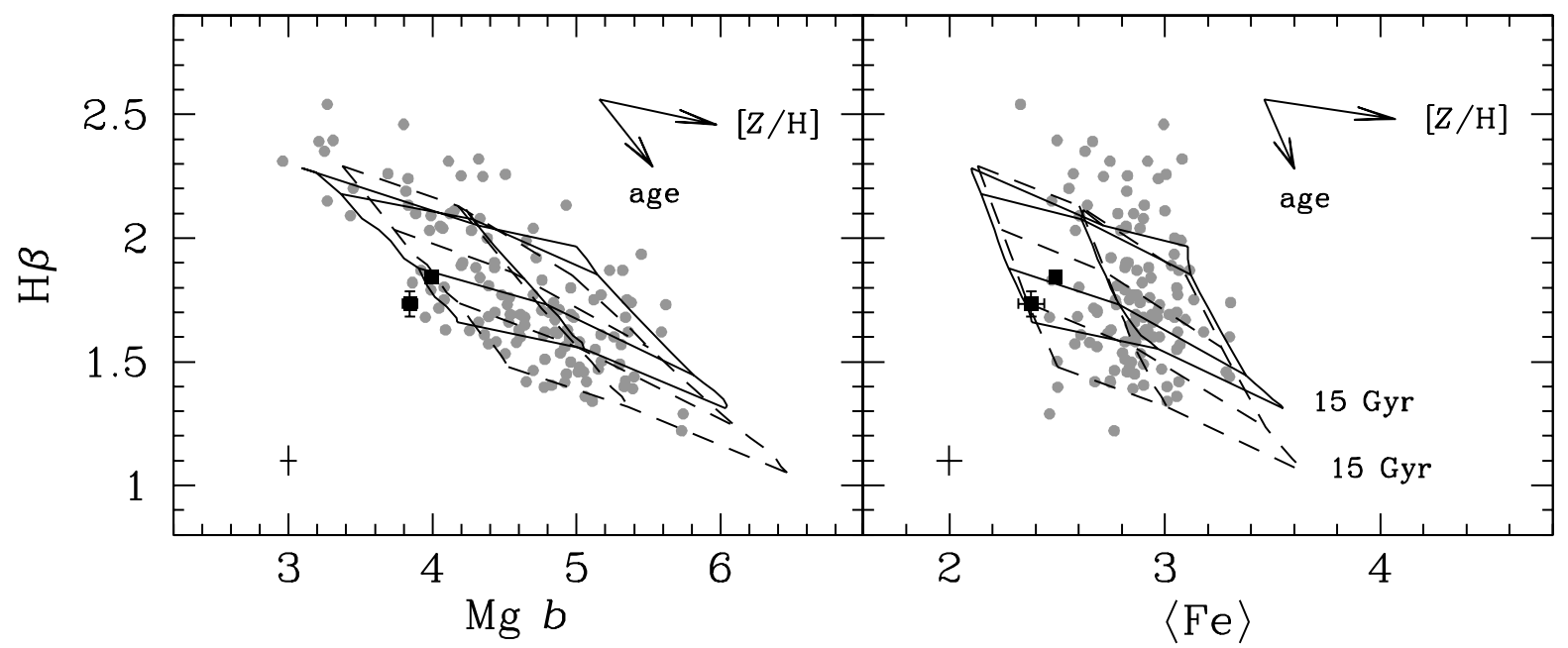

Fig. 2. $\mathrm{Mg} b$ and $\langle\mathrm{Fe}\rangle$ vs. $\mathrm{H} \beta$ for SSP models of constant ages $t=3,5,10,15 \mathrm{Gyr}$ and metallicities $[Z / \mathrm{H}]=0.0,0.35,0.67$. The dashed and solid lines are Model 1 and Model 2, respectively (Table 1). Grey circles are early-type galaxy data measured within $0.1 r_{\mathrm{e}}$ (González 1993; Mehlert et al. 2000; Beuing et al. 2002). The typical error is given in the lower-left corner. Black squares are the Bulge clusters NGC 6528 and NGC 6553 (Puzia et al. 2002).

suggests that the Red Giant Branch of the Salasnich et al. (2000) $\alpha /$ Fe enhanced tracks is indeed too warm (Schiavon et al. 2002). The integrated absorption indices of the two Bulge clusters NGC 6528 and NGC 6553 are consistent with both $\alpha /$ Fe enhanced and solar-scaled stellar tracks, as discussed before. At metallicities above solar, where the impact of $\alpha / \mathrm{Fe}$ enhanced stellar tracks becomes most significant and the ages of ellipticals become alarmingly high, globular clusters are not available. We are trapped in the usual dilemma for the stellar population models of elliptical galaxies. Hence, further investigations on the effect of $\alpha / \mathrm{Fe}$ enhanced mixtures on stellar evolutionary tracks at super-solar metallicities would be very valuable.

\section{References}

Beuing, J., Bender, R., Mendes de Oliveira, C., Thomas, D., \& Maraston, C. 2002, A\&A, 395, 431

Cassisi, S., Castellani, M., \& Castellani, V. 1997, A\&A, 317, 10

Ferreras, I., Melchiorri, A., \& Silk, J. 2001, ApJ, 327, L47

González, J. 1993, Ph.D. Thesis, University of California, Santa Cruz

Kim, Y.-C., Demarque, P., Yi, S. K., \& Alexander, D. R. 2002, ApJS, 143,499

Knox, L., Christensen, N., \& Skordis, C. 2001, ApJ, 563, 95
Lauberts, A., \& Valentijn, E. A. 1989, The Surface Photometry Catalogue of the ESO-Upsalla Galaxies (Garching: ESO)

Maraston, C. 1998, MNRAS, 300, 872

Maraston, C., Greggio, L., Renzini, A., et al. 2003, A\&A, 400, 823

Mehlert, D., Saglia, R. P., Bender, R., \& Wegner, G. 2000, A\&AS, 141,449

Perlmutter, S., Aldering, G., Gddhaber, G., et al. 1999, ApJ, 517, 565

Puzia, T., Saglia, R. P., Kissler-Patig, M., et al. 2002, A\&A, 395, 45

Riess, A. G., Filippenko, A. V., Challis, P., et al. 1998, AJ, 116, 1009

Salaris, M., Chieffi, A., \& Straniero, O. 1993, ApJ, 414, 580

Salaris, M., \& Weiss, A. 1998, A\&A, 335, 943

Salasnich, B., Girardi, L., Weiss, A., \& Chiosi, C. 2000, A\&A, 361, 1023

Schiavon, R., Faber, S. M., Castilho, B. V., \& Rose, J. A. 2002, ApJ, 580,850

Thomas, D., Maraston, C., \& Bender, R. 2002, in Rev. Mod. Astron., 15, Astronomy with Large Telescopes from Ground and Base, ed. R. E. Schielicke (Berlin: Wiley-VCH), 219 [astro-ph/0202166]

Thomas, D., Maraston, C., \& Bender, R. 2003, MNRAS, 339, 897

Trager, S. C., Faber, S. M., Worthey, G., \& González, J. J. 2000, AJ, 119,164

Tripicco, M. J., \& Bell, R. A. 1995, AJ, 110, 3035

VandenBerg, D. A., Swenson, F. J., Rogers, F. J., Iglesias, C. A., \& Alexander, D. R. 2000, ApJ, 532, 430

Weiss, A., Peletier, R. F., \& Matteucci, F. 1995, A\&A, 296, 73

Worthey, G., Faber, S. M., \& González, J. J. 1992, ApJ, 398, 69 